\title{
Influence on interfacial diffusive scatterings of the angle between the magnetizations of the two magnetic layers in a magnetic trilayer
}

\author{
Sui-Pin Chen ${ }^{\mathrm{a}}$, Ching-Ray Chang ${ }^{\mathrm{b}, *}$, Chih-Huang Lai ${ }^{\mathrm{c}}$ \\ ${ }^{a}$ Department of Applied Physics, National Chia Yi University, 600 Chia Yi, Taiwan \\ ${ }^{\mathrm{b}}$ Department of Physics, National Taiwan University, 10617 Taipei, Taiwan \\ ${ }^{\mathrm{c}}$ Department of Materials Science and Engineering, National Tsing Hua University, 300 Hsinchu, Taiwan
}

Available online 16 November 2006

\begin{abstract}
To understand the influence of the relative angle between the magnetizations of the two magnetic layers in a magnetic trilayer on diffusive scatterings of a transport electron, we study an A-B-C magnetic trilayer by use of the effective mean-free-path model. Our theoretical results indicate that varying the relative angle can enhance or suppress an interfacial diffusive scattering on a transport spindependent electron if the thickness of the metallic layer is smaller than the spin relaxation length.
\end{abstract}

(C) 2006 Elsevier B.V. All rights reserved.

PACS: 73.40; 73.61; 75.70; 73.50.G

Keywords: Boltzmann transport equation; Effective mean-free-path model; Diffusive scattering; Magntic trilayer

The effective mean-free-path model, which is equivalent to the Boltzmann transport equation model, is useful to study the electron transport in a finite system [1]. Given boundary conditions, the solution of the electron distribution to specify the electron transport can be easily obtained from this model, and is always explicitly expressed in an analytic form.

The electron distribution $f_{\mathbf{v}}^{\sigma}(\mathbf{r})$, corresponding to the energy $\varepsilon$ and the velocity $\mathbf{v}$ at the position $\mathbf{r}$, can be divided into two parts: the Fermi-Dirac distribution $f^{0, \sigma}(\varepsilon)$ and $g_{\mathbf{v}}^{\sigma}(\mathbf{r})$, such that $f_{\mathbf{v}}^{\sigma}(\mathbf{r})=f^{0, \sigma}(\varepsilon)+g_{\mathbf{v}}^{\sigma}(\mathbf{r})$, where the spin index $\sigma$ is $+1(-1,0)$ for spin-up (spin-down, spin-independent) state [1-3]. The spin-dependent Fermi-Dirac distribution is $f^{0, \sigma}(\varepsilon)=1 /\left[\exp \left[\beta\left(\varepsilon-\mu^{\sigma}\right)\right]+1\right]$, where the Boltzmann constant $k_{\mathrm{B}}$ and the temperature $T$ are related by $\beta=1 / k_{\mathrm{B}} T$. The energy of an electron is $\varepsilon=1 / 2 m v^{2}$, where $m$ is the mass of an electron. The spin-dependent chemical potential $\mu^{\sigma}$ and the spin-independent chemical potential $\mu^{0}$ are related by $\mu^{\sigma}=\mu^{0}+h$, where $h$ is the molecular field of a magnetic material. The $g_{\mathbf{v}}^{\sigma}(\mathbf{r})$ originates from all diffusive boundary effects under the electric field, and is

\footnotetext{
*Corresponding author. Tel.: + 886233665188 ; fax: 886223639984.

E-mail address: crchang@phys.ntu.edu.tw (C.-R. Chang).
}

$g_{\mathbf{v}}^{\sigma}(\mathbf{r})=\left[-\frac{\partial f^{0, \sigma}(\varepsilon)}{\partial \varepsilon}\right]\left[-\mathbf{e}_{\mathbf{v}} \cdot e \mathbf{E} \lambda^{\sigma}+e \mu_{\mathbf{v}}^{\sigma}(\mathbf{r})\right]$

where $\mathbf{e}_{\mathbf{v}}$ is a unit vector in the transport direction, $\lambda^{\sigma}=$ $v_{\mathrm{F}}^{\sigma} \times \tau^{\sigma}$ is the mean free path, $\tau^{\sigma}$ is the relaxation time, $-e$ is the electron charge, and $\mathbf{E}$ is the applied uniform electric field. The term $\mu_{\mathbf{v}}^{\sigma}(\mathbf{r})$ can be used to specify the charge accumulation in a system.

In the effective mean-free-path model, $\mu_{\mathbf{v}}^{\sigma}(\mathbf{r})$ is expressed as

$\mu_{\mathbf{v}}^{\sigma}(\mathbf{r})=\mathbf{e}_{\mathbf{v}} \cdot e \mathbf{E} \lambda^{\sigma} \times D_{\text {equ, }}^{\sigma} \times \exp \left[\frac{-\left|\mathbf{r}-\mathbf{r}_{\mathrm{b}, \mathbf{v}}\right|}{\lambda^{\sigma}}\right]$,

where $D_{\text {equ,v }}^{\sigma}$ is the equilibrium diffusion parameter and $\mathbf{r}_{\mathrm{b}, \mathbf{v}}$ is the position of the boundary [1]. The same position of $\mathbf{r}_{\mathrm{b}, \mathbf{v}}$ is divided into $\mathbf{r}_{\mathbf{v}}$ and $\mathbf{r}_{-\mathbf{v}}$ for the transport electron along $\mathbf{e}_{-\mathbf{v}}$ and $\mathbf{e}_{\mathbf{v}}$, respectively. The only unknown $D_{\text {equ,v }}^{\sigma}$ can be determined by use of injecting an incident inverse-transport electron into the system [1]. This unit incident inversetransport electron is along the transport direction $-\mathbf{e}_{\mathbf{v}}=$ $\mathbf{e}_{-\mathbf{v}}$ and is injected from $\mathbf{r}=\mathbf{r}_{-\mathbf{v}}$. The value of $D_{\text {equ,v }}^{\sigma}$ is associated with the superposition of all effective diffusive scatterings on this electron, and can thus be obtained. 
The effective diffusive scattering can be specified by the diffusion parameters $D^{\sigma}$ of the diffusive boundary and the mean free paths $\lambda^{\sigma}$ in a system [1]. At the diffusive boundary, the relation among the diffusion parameter $D^{\sigma}$, the reflection parameter $R^{\sigma}$, and the transmission parameter $T^{\sigma}$ is given by $R^{\sigma}+T^{\sigma}=1-D^{\sigma}=S^{\sigma}$. The $S^{\sigma}$ denotes the degree of diffuseness of the scattering [4,5]. For a single diffusive boundary, the effective diffusive scattering on the transport electron depends on $D^{\sigma}$ and decays exponentially with the distance between the diffusive boundary and the transport electron [1]. The decaying length of the effective diffusive scattering is characterized by $\lambda^{\sigma}$. In structures with more than one diffusive boundary, the determination to specify all effective diffusive scatterings is associated with the sum of their individual effective diffusive scatterings from all diffusive boundaries [1]. Besides, the diffusion parameter $D_{\mathrm{A}-\mathrm{B}}^{\sigma}$ at the $\mathrm{A}-\mathrm{B}$ interface will be modified as $D_{\mathrm{A} \rightarrow \mathrm{B}}^{\prime \sigma}\left(D_{\mathrm{B} \rightarrow \mathrm{A}}^{\prime \sigma}\right)$, which specifies the diffusive scattering from the $\mathrm{A}-\mathrm{B}$ interface on a transport electron at the A-B interface when this electron without spin flip scattering tends to transport along the direction from the A (B) layer through the A-B interface to the B (A) layer $[1,6]$. The modified diffusion parameters $D_{\mathrm{A} \rightarrow \mathrm{B}}^{\prime \sigma}$ and $D_{\mathrm{B} \rightarrow \mathrm{A}}^{\prime \sigma}$ are given by

$D_{\mathrm{A} \rightarrow \mathrm{B}}^{\prime \sigma}=1-R_{\mathrm{A} \rightarrow \mathrm{B}}^{\sigma}-T_{\mathrm{A} \rightarrow \mathrm{B}}^{\sigma}$,

$D_{\mathrm{B} \rightarrow \mathrm{A}}^{\prime \sigma}=1-R_{\mathrm{B} \rightarrow \mathrm{A}}^{\sigma}-T_{\mathrm{B} \rightarrow \mathrm{A}}^{\sigma}$,

where $R_{\mathrm{A} \rightarrow \mathrm{B}}^{\sigma}=R_{\mathrm{A}-\mathrm{B}}^{\sigma}, \quad R_{\mathrm{B} \rightarrow \mathrm{A}}^{\sigma}=R_{\mathrm{A}-\mathrm{B}}^{\sigma}, \quad T_{\mathrm{A} \rightarrow \mathrm{B}}^{\sigma}=\lambda_{\mathrm{B}}^{\sigma} / \lambda_{\mathrm{A}}^{\sigma} \times$ $T_{\mathrm{A}-\mathrm{B}}^{\sigma}$, and $T_{\mathrm{B} \rightarrow \mathrm{A}}^{\sigma}=\lambda_{\mathrm{A}}^{\sigma} / \lambda_{\mathrm{B}}^{\sigma} \times T_{\mathrm{A}-\mathrm{B}}^{\sigma}$.

In this paper, we study an A-B-C trilayer with two infinite planes. The $\mathrm{A}$ magnetic layer and the $\mathrm{C}$ magnetic layer are separated by the $\mathrm{B}$ metallic layer. The thickness of the $\mathrm{B}$ layer is $d_{\mathrm{B}}$, the $\mathrm{A}-\mathrm{B}(\mathrm{B}-\mathrm{C})$ interface is at $z=0$ $\left(z=d_{\mathrm{B}}\right)$, and the relative angle between the magnetizations of the $\mathrm{A}$ and the $\mathrm{C}$ layers is $\theta$. The effect of $\theta$ on the diffusive scatterings of a transport electron is understood using $D_{\text {equ,B, }>}^{\sigma}$. The $>$ is used to represent to $\mathbf{v}$ toward the forward boundary. The term $D_{\text {equ,B, }>}^{\sigma}$ specifies the effective diffusive scattering on the electron at $z=0$ of the B layer tending to transport along the $\mathrm{C}$ layer, and its value can be determined by using the effective mean-free-path model [1]. For simplicity, the $\mathrm{C}$ layer is assumed to be the same as the A layer, and the value of $D_{\text {equ,B, }>}^{\sigma}$ is then

$$
\begin{aligned}
& D_{\text {equ,B, }>}^{\sigma} \\
& =\frac{D_{(1)}^{\sigma}+D_{(2)}^{\sigma}+\left(L_{\mathrm{B}}^{\sigma \Rightarrow-\sigma} /\left(1-L_{\mathrm{B}}^{-\sigma \Rightarrow-\sigma}\right)\right) \times\left[D_{(1)}^{-\sigma}+D_{(2)}^{-\sigma}\right]}{1-L_{\mathrm{B}}^{\sigma \Rightarrow \sigma}-\left(\left(L_{\mathrm{B}}^{\sigma \Rightarrow-\sigma} \times L_{\mathrm{B}}^{-\sigma \Rightarrow \sigma}\right) /\left(1-L_{\mathrm{B}}^{-\sigma \Rightarrow-\sigma}\right)\right)}, \\
& D_{(1)}^{ \pm \sigma}=D_{\mathrm{B} \rightarrow \mathrm{A}}^{ \pm \sigma \sigma}, \\
& D_{(2)}^{ \pm \sigma}=R_{\mathrm{B} \rightarrow \mathrm{A}}^{ \pm \sigma} \times t_{\mathrm{B}} \times F^{ \pm \sigma \rightarrow+\sigma} \times D_{\mathrm{B} \rightarrow \mathrm{A}}^{\prime+\sigma} \\
& +R_{\mathrm{B} \rightarrow \mathrm{A}}^{ \pm \sigma} \times t_{\mathrm{B}} \times F^{ \pm \sigma \rightarrow-\sigma} \times D_{\mathrm{B} \rightarrow \mathrm{A}}^{\prime-\sigma},
\end{aligned}
$$

$$
\begin{aligned}
L_{\mathrm{B}}^{+\sigma \Rightarrow \pm \sigma}= & R_{\mathrm{B} \rightarrow \mathrm{A}}^{+\sigma} \times t_{\mathrm{B}} \times F^{+\sigma \rightarrow+\sigma} \times R_{\mathrm{B} \rightarrow \mathrm{C}}^{+\sigma} \times t_{\mathrm{B}} \times F^{+\sigma \rightarrow \pm \sigma} \\
& +R_{\mathrm{B} \rightarrow \mathrm{A}}^{+\sigma} \times t_{\mathrm{B}} \times F^{+\sigma \rightarrow-\sigma} \times R_{\mathrm{B} \rightarrow \mathrm{C}}^{-\sigma} \times t_{\mathrm{B}} \times F^{-\sigma \rightarrow \pm \sigma},
\end{aligned}
$$$$
t_{\mathrm{B}}=\exp \left[\frac{-d_{\mathrm{B}} / \cos \left(\mathbf{e}_{\mathbf{v}} \cdot \mathbf{e}_{z}\right)}{\lambda_{\mathrm{B}}}\right] .
$$

The terms $F^{\sigma \rightarrow \sigma}=\cos ^{2}(\theta / 2)$ and $F^{\sigma \rightarrow-\sigma}=\sin ^{2}(\theta / 2)$ are the probabilities for an electron of spin $\sigma$ (with respect to the magnetization in the A layer) at $z=d_{\mathrm{B}} / 2$ to continue as spin $\pm \sigma$ (with respect to the magnetization in the $\mathrm{C}$ layer) at $z=d_{\mathrm{B}} / 2$, respectively [2].

The $D_{\text {equ,B, }>}^{\sigma}$ in Eq. (4a) returns to the original $D_{\mathrm{A}-\mathrm{B}}^{\sigma}$ when $d_{\mathrm{B}} \rightarrow \infty$ and $\lambda_{\mathrm{A}}^{\sigma}=\lambda_{\mathrm{B}}$. If $d_{\mathrm{B}} \rightarrow \infty$, the diffusive effect from the $\mathrm{B}-\mathrm{C}$ interface on the transport electron at the $\mathrm{A}-\mathrm{B}$ interface can reasonably be ignored; if $\lambda_{\mathrm{A}}^{\sigma}=\lambda_{\mathrm{B}}$, no asymmetry exists between the transport properties. Moreover, $D_{\text {equ,B, }>}^{\sigma}$ in Eq. (4a) is modified by $D_{\mathrm{B} \rightarrow \mathrm{A}}^{\prime \sigma}$ in Eq. (3a) for $d_{\mathrm{B}} \rightarrow \infty$ but $\lambda_{\mathrm{A}}^{\sigma} \neq \lambda_{\mathrm{B}}$, indicating that the asymmetry of $\lambda_{\mathrm{A}}^{\sigma} \neq \lambda_{\mathrm{B}}$ can induce a diffusive scattering at the $\mathrm{A}-\mathrm{B}$ interface [1,6]. It is evident in Eq. (4a) that $D_{\text {equ,B, }}^{\sigma}$. depends on the relative angle $\theta$ and on the thickness $d_{\mathrm{B}}$. This $D_{\text {equ }, \mathrm{B},>}^{\sigma} \neq D_{\mathrm{B} \rightarrow \mathrm{A}}^{\sigma} \neq D_{\mathrm{B}-\mathrm{A}}^{\sigma}$ can be interpreted as one specifying the effective diffusive scattering from the $\mathrm{A}-\mathrm{B}$ interface on a transport electron, when this electron tends to transport along the direction from the $\mathrm{B}$ layer to the A layer, since $\lambda_{\mathrm{A}}^{\sigma} \neq \lambda_{\mathrm{B}}[1,6], \quad \theta \neq 0$, and $d_{\mathrm{B}} \neq \infty$. Furthermore, Eq. (4a) for $D_{\mathrm{A}-\mathrm{B}}^{+}=D_{\mathrm{B}-\mathrm{C}}^{+}<D_{\mathrm{A}-\mathrm{B}}^{-}=D_{\mathrm{B}-\mathrm{C}}^{-}$ indicates that $\theta$ can enhance but suppress interfacial diffusive scatterings on spin-up and spin-down electrons, respectively. Under this boundary condition, $\theta$ suppresses the asymmetry of $D_{\text {equ,B, },}^{-} / D_{\text {equ,B, }>}^{+}$. In contrast, if $D_{\mathrm{A}-\mathrm{B}}^{+}<D_{\mathrm{A}-\mathrm{B}}^{-}$but $D_{\mathrm{B}-\mathrm{C}}^{+}>D_{\mathrm{B}-\mathrm{C}}^{-}, \theta$ enhances the asymmetry of $D_{\text {equ,B, }>}^{-} / D_{\text {equ,B, }>}^{+}$. In summary, the relative angle between the magnetizations of the two magnetic layers in a magnetic trilayer can enhance or suppress an interfacial diffusive scattering on transport spin-dependent electrons, depending on the given boundary conditions, if the thickness of metallic layer is smaller than the spin relaxation length.

This research was supported by Ministry of Economic Affairs of Taiwan under Grants 95-EC-17-A-08-S1-0006 and by the National Science Council under Grant No NSC 95-2745-M-415-001.

\section{References}

[1] S.-P. Chen, C.-R. Chang, Phys. Rev. B 72 (2005) 064445.

[2] R.E. Camley, J. Barnas, Phys. Rev. Lett. 63 (1989) 664.

[3] T. Valet, A. Fert, Phys. Rev. B 48 (1993) 7099.

[4] R.Q. Hood, L.M. Falicov, Phys. Rev. B 46 (1992) 8287.

[5] P. Rennert, A. Brzezinski, Phys. Rev. B 52 (1995) 1612.

[6] S.-P. Chen, C.-R. Chang, J. Appl. Phys. 99 (2006) 08R502. 